\title{
Long-term fluorescence lifetime imaging of a genetically encoded sensor for caspase-3 activity in mouse tumor xenografts
}

Victoria Zherdeva

Natalia I. Kazachkina

Vladislav Shcheslavskiy

Alexander P. Savitsky 


\title{
Long-term fluorescence lifetime imaging of a genetically encoded sensor for caspase-3 activity in mouse tumor xenografts
}

\author{
Victoria Zherdeva, ${ }^{a}$ Natalia I. Kazachkina, ${ }^{a}$ Vladislav Shcheslavskiy, ${ }^{b}$ and Alexander P. Savitsky ${ }^{a, *}$ \\ ${ }^{a}$ Research Center of Biotechnology of the Russian Academy of Sciences, Bach Institute of Biochemistry, Moscow, Russia \\ ${ }^{\mathrm{b}}$ Becker \& Hickl GmbH, Berlin, Germany
}

\begin{abstract}
Caspase-3 is known for its role in apoptosis and programmed cell death regulation. We detected caspase-3 activation in vivo in tumor xenografts via shift of mean fluorescence lifetimes of a caspase-3 sensor. We used the genetically encoded sensor TR23K based on the red fluorescent protein TagRFP and chromoprotein KFP linked by 23 amino acid residues (TagRFP-23-KFP) containing a specific caspase cleavage DEVD motif to monitor the activity of caspase-3 in tumor xenografts by means of fluorescence lifetime imaging-Forster resonance energy transfer. Apoptosis was induced by injection of paclitaxel for A549 lung adenocarcinoma and etoposide and cisplatin for HEp-2 pharynx adenocarcinoma. We observed a shift in lifetime distribution from 1.6 to $1.9 \mathrm{~ns}$ to 2.1 to $2.4 \mathrm{~ns}$, which indicated the activation of caspase-3. Even within the same tumor, the lifetime varied presumably due to the tumor heterogeneity and the different depth of tumor invasion. Thus, processing time-resolved fluorescence images allows detection of both the cleaved and noncleaved states of the TR23K sensor in real-time mode during the course of several weeks noninvasively. This approach can be used in drug screening, facilitating the development of new anticancer agents as well as improvement of chemotherapy efficiency and its adaptation for personal treatment. $\odot$ The Authors. Published by SPIE under a Creative Commons Attribution 3.0 Unported License. Distribution or reproduction of this work in whole or in part requires full attribution of the original publication, including its DOI. [DOI: 10.1117/1.JBO.23.3.035002]
\end{abstract}

Keywords: FLIM-FRET; genetically encoded sensor; caspase-3; red fluorescent proteins; tumor xenografts; small animal imaging; drug screening.

Paper 170693R received Oct. 25, 2017; accepted for publication Feb. 1, 2018; published online Mar. 2, 2018.

\section{Introduction}

Continuous and recurrent noninvasive real-time monitoring of cellular and molecular events in whole organisms is the main paradigm of fluorescence molecular imaging. It can be achieved using different types of fluorescent probes such as exogenous fluorophores, genetically encoded probes, and intrinsic fluorophores as well as employing specialized visualization techniques.

Exogenous probes such as near-infrared fluorescent probes, ${ }^{1,2}$ nanoparticles (quantum dots, ${ }^{3}$ upconverting nanophosphors, ${ }^{4}$ and multimodal nanoparticles ${ }^{5}$ ) are administered exogenously and the pharmacokinetics of these probes needs to be considered separately from the pharmacokinetics of potential drugs during the measurements. Despite the fact that genetically encoded bioluminescent luciferase-luciferin reporters have extremely high sensitivity, ${ }^{6}$ while phytochromes (iRFP) are suitable for deep located tumor imaging, ${ }^{7,8}$ the former require luciferin injection while the latter need other external biliverdin-chromophore inoculation or fixed level of endogenous biliverdin.

The serious advantage of fluorescent proteins (FPs) is their applicability for long-term in vivo measurements of potential drug pharmacokinetics without crosstalk with labeling methods.

FPs are widely used as genetically encoded cellular or subcellular probes ${ }^{10-13}$ in small animal fluorescence imaging

*Address all correspondence to: Alexander P. Savitsky, E-mail: apsavitsky@ inbi.ras.ru
$(\mathrm{SAFI})^{14,15}$ based on the genetically engineered mice and xenografts. ${ }^{16,17}$ FPs are also used for Forster resonance energy transfer (FRET) experiments. ${ }^{18-21}$ In addition to the bluegreen $^{22}$ and cyan-yellow FRET pairs, ${ }^{23,24}$ red and far-red FRET pairs have also became available. ${ }^{14,15,25}$ Using red FPs allows working at longer wavelengths that results in the reduced light scattering (deeper penetration depth) and reduced autofluorescence. $^{26}$

The attractive targets for SAFI are the number of proteases such as caspases, metalloproteinases, cathepsins, and the urokinase plasminogen activator. ${ }^{1,27}$ Proteases are involved in the pathogenesis of different types of diseases such as cardiovascular and inflammatory diseases, osteoporosis, neurological disorders, and cancer. ${ }^{28}$ In experimental animal models, the enzymatic activity often needs to be monitored up to several weeks. A number of FP-based genetically encoded sensors are best suited for these purposes, ${ }^{12,18}$ and caspase- 3 biosensors are the most common among them. ${ }^{18}$ Caspase-3 is well known for its role in performing the specific cleavage of many cellular proteins in programmed cell death, as well as in regulation of cellular homeostasis. $^{28-30}$

Typically, an amino acid sequence that can be specifically recognized and cleaved by the enzyme of interest is placed between two FPs - a donor and an acceptor for protease FRET-sensors. ${ }^{18}$ Cleavage of a substrate by the protease leads to a change in FRET efficiency which results in a change of donor fluorescence intensity and lifetime. An important property of these genetically encoded substrates is the resilience 
of the FPs themselves to proteases. ${ }^{31}$ It has been shown that FRET efficiency of FP-based sensors does not change upon activation of proteases that are nonspecific to caspase-3 target amino acids sequence DEVD. ${ }^{32}$

Steady-state and time-resolved measurements are commonly used with FRET-sensor readouts. ${ }^{18,33}$ FP-based FRET-sensors have most often been used for detection of caspase- 3 activation by steady-state techniques in target cells in vitro. ${ }^{34-38}$ The genetically encoded sensor SCAT3, based on ECFP and Venus, expressed in transgenic mice has been used for ratiometric visualization of apoptosis in living mammalian tissues. ${ }^{39}$

In steady-state techniques, the variation of donor and acceptor concentrations in the biological sample due to differences in FP maturation speed and photobleaching must be taken into account. Donor crosstalk with the acceptor emission band and direct excitation of the acceptor molecules lead to inaccuracies in the measurement of acceptor intensities, therefore careful separate calibration of the donor and acceptor is required. Some optimized steady-state techniques for FP FRET readout such as two-channel imaging with an algorithm of excitation and emission crosstalk correction, acceptor photobleaching, spectral imaging, and fluorescence polarization imaging have been used. ${ }^{18,21,24}$

Until recently, planar [two-dimensional (2-D)-imaging] ${ }^{16-18}$ and tomography (three-dimensional-imaging) ${ }^{40-44}$ have been the most widespread methods for in vivo studies. ${ }^{45-47}$ The incorporation of more advanced fluorescence techniques such as FRET-FLIM,${ }^{19,48-50}$ fluorescence recovery after photobleaching, as well as photoswitching/photoactivation techniques into imaged-based drug discovery platforms has provided a new approach to real-time dynamic visualization both in vitro and in vivo. ${ }^{51}$

There are a number of reports devoted to the methodology for development of a practical FRET experiment in vivo using FLIM. FLIM allows discrimination of two different red FPs in subcutaneous mouse models. ${ }^{52}$ Fluorescence lifetime imagingForster resonance energy transfer (FLIM-FRET) techniques are based on the measurement of the fluorescence lifetime of the donor in the FRET pair. This method overcomes the problems of fluctuation of fluorophore concentration in biological samples and does not require a donor calibration procedure. ${ }^{53-56}$ In addition, FLIM-FRET approach, compared to intensity-based methods, is ideal for the cases when acceptors with low quantum yield or completely dark are used. ${ }^{57}$ A xenograft tumor model expressing GFP coupled to a chemokine receptor was analyzed by imaging deep-tissue multiphoton fluorescence lifetime in vivo ${ }^{58}$ FLIM was also used to image a caspase-3-based FRET probe to examine the development of drug-resistance in a syngeneic mouse tumor model ex vivo and on re-isolated cells from metastatic tissue. ${ }^{59}$ Spatial mapping of FRET among genetically encoded FPs in live mice using lifetime tomography has also been demonstrated. ${ }^{33}$ SNARE assembly has been visualized using two-photon fluorescence lifetime imaging approaches in both live and chemically fixed tissues. ${ }^{60}$ FLIM combined with optical projection tomography has also been used to observe apoptosis in live zebrafish. ${ }^{61}$

Earlier we used FLIM-FRET for detection of caspase-3 activation in tumor cells in vitro. ${ }^{52,53}$ This paper describes single photon FLIM-FRET of a genetically encoded sensor for caspase-3 imaging in vivo in mouse tumor xenografts generated from A549 and HEp-2 cells. We demonstrate that this system allows evaluation of chemotherapeutic intervention efficiency toward tumor cells within an animal model.

\section{Materials and Methods}

\subsection{FRET-Sensor TR23K}

We use a genetically encoded caspase-3 FRET-sensor TR23K based on a pair of FPs-TagRFP as a donor and nonfluorescent chromoprotein KFP as an acceptor, which are connected by a flexible amino acid linker GTGGSGGDEVDGTGGSGDPPVAT $^{52,53}$ [Fig. 1(a)]. Normalized absorption and emission spectra are presented in Fig. 1(b). Spectral overlap integral for TagRFP-KFP is significantly higher than that for similar proteins (Table 1). Because KFP is almost nonfluorescent, ${ }^{62}$ there is no spectral crosstalk of donor and acceptor, and it is

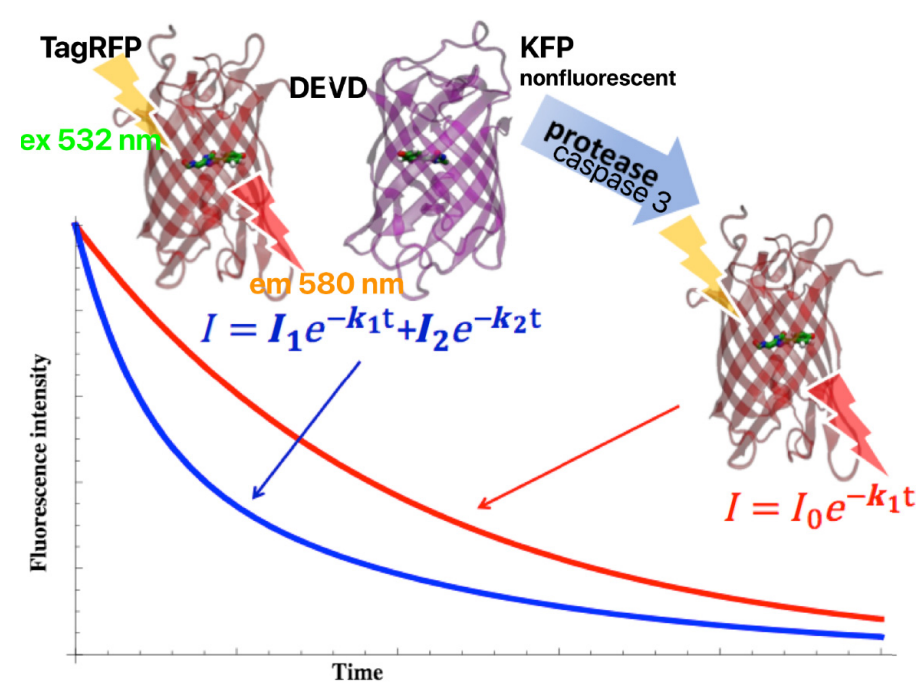

(a)

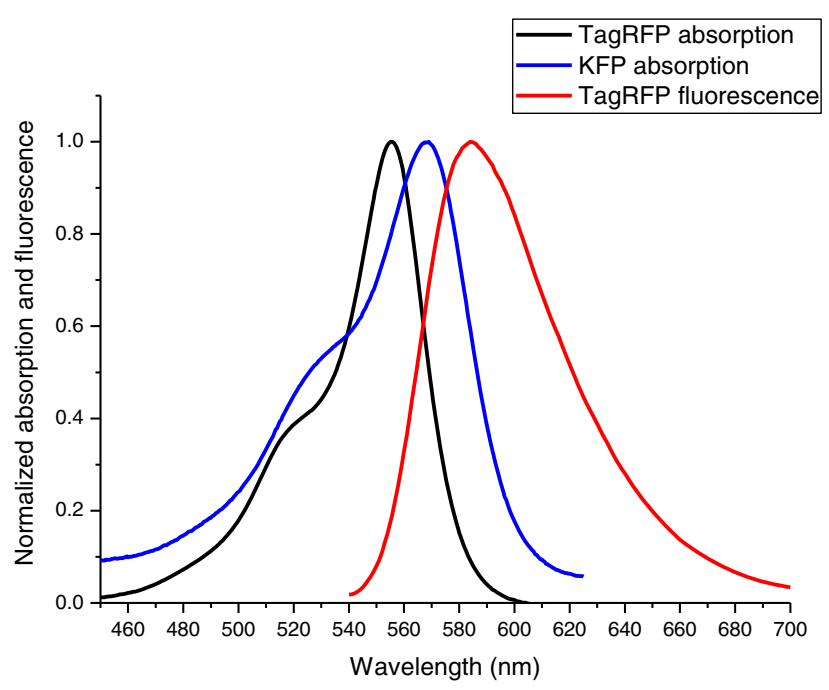

(b)

Fig. 1 (a) Schematic representation of FRET-sensor TR23K. (b) Normalized absorption spectra of TagRFP and KFP and fluorescence spectra for TagRFP. KFP is nonfluorescent. 
Table 1 Spectral overlap integrals for FRET pair of FPs.

\begin{tabular}{lc} 
Donor $\rightarrow$ Acceptor & $\varphi_{\mathrm{D}} \cdot \mathrm{J}, \mathrm{nm}^{4} \cdot-1 \cdot \mathrm{cm}^{-1}$ \\
TagRFP $\rightarrow$ KFP & $2.39 \cdot 10^{15}$ \\
TagGFP $\rightarrow$ TagRFP & $1.91 \cdot 10^{15}$ \\
EGFP $\rightarrow$ EYFP & $1.57 \cdot 10^{15}$ \\
EYFP $\rightarrow$ DsRed & $7.19 \cdot 10^{14}$ \\
ECFP $\rightarrow$ EYFP & $4.60 \cdot 10^{14}$ \\
\hline
\end{tabular}

possible to detect FRET efficiency based on fluorescence lifetime of the donor protein-TagRFP. The DEVD tetrapeptide is embedded in the linker and specifically recognized by caspase- 3 and cleaved, leading to the increase of TagRFP fluorescence intensity and lifetime. ${ }^{52}$ The use of the latter parameter is more convenient because it is independent of such factors as donor photobleaching and concentration of the sensor. Despite the tetrameric structure of TagRFP-23-KFP sensor, the DEVD site in the linker is located far enough from the barrels of TagRFP and KFP to be cleaved by caspase- 3 without steric hindrances. ${ }^{53,57}$

\subsection{Cell Culture, Transfection, and Treatment of Tumor Cells}

Tumor cell lines of lung adenocarcinoma A549 and larynx carcinoma HEp-2 originating from the ATCC were obtained from the Russian Cell Collection (St. Petersburg, Russia). Cells were cultured in complete RPMI-1640 (Paneco, Russia) medium with $10 \%$ fetal bovine (Bioclot, SA), penicillin (100 units $/ \mathrm{ml})$, and streptomycin $(100 \mathrm{mg} / \mathrm{ml})$ in $5 \% \mathrm{CO}_{2}$ at $37^{\circ} \mathrm{C}$ in a humidified incubator.

The plasmid pTR23K contains encodes TagRFP and KFP in a single reading frame with a linker sequence encoding 23 amino acids. $^{51}$

Transfection of A549 and HEp-2 cell line was carried out using the pLV lentivector system (Evrogen, Russia). The obtained pLVTR23K plasmid contains TagRFP-23-KFP gene with codon usage optimized for high expression in mammalian cells. The titer of viral particles was estimated at $10^{5} \mathrm{~T} . \mathrm{U} / \mathrm{ml}$. One day before transfection, cells were plated in six-well plates at $1 \times 10^{4}$ in $2 \mathrm{ml}$ of growth medium RPMI-1640 containing 5\% fetal bovine serum so that cells would be $70 \%$ confluent the time of transduction. Near-confluent A549 cells were incubated with the threefold excess of viral particles for $24 \mathrm{~h}$ before being replenished with fresh medium. The cells were examined on an inverted fluorescence microscope $48 \mathrm{~h}$ post-transduction. The cells were grown in complete RPMI-1640 (Paneco, Russia) medium with $10 \%$ fetal bovine (Bioclot, SA) serum for 10 passages without losing their fluorescent properties.

For detection of activation of caspase- 3 in vitro after treatment with anticancer drugs cells were seeded on a $\mu$-Dish $35 \mathrm{~mm}$ (ibidi $\mathrm{GmbH}$, Germany) and incubated in $5 \% \mathrm{CO}_{2}$ at $37^{\circ} \mathrm{C}$ in a humidified incubator. After $48 \mathrm{~h}$ of growing paclitaxel (Lance, Russia) were added in concentration of $30 \mu \mathrm{g} / \mathrm{ml}$ to the A549-TR23K and incubated for $24 \mathrm{~h}$. After $48 \mathrm{~h}$ of growing a combination of etoposide (Teva, Israel)/cisplatin (Teva, Israel) were added to the HEp-2-TR23K in concentration of $200 / 10 \mu \mathrm{g} / \mathrm{ml}$ and incubated for $24 \mathrm{~h}$. Posttreatment caspase- 3 activation was visualized on time-resolved confocal fluorescence microscope.

\subsection{FLIM-FRET Microscopy}

FLIM-FRET of cells was performed using the time-resolved fluorescence confocal microscope MicroTime 200 (PicoQuant $\mathrm{GmbH}$, Berlin, Germany). Fluorescence was excited by 532-nm picosecond diode laser PicoTA (PicoQuant $\mathrm{GmbH}$, Berlin, Germany) (repetition rate and pulse-width are $80 \mathrm{MHz}$ and $100 \mathrm{ps}$, respectively). The fluorescence signal was selected by a narrow bandpass filter (BP580/25, Chroma) and detected by a single-photon counting module (SPCM-AQRH, Perkin Elmer, Canada). Olympus (UPlanSApo $60 \times / 1.35$ ) has been used in all measurements. Cells were seeded on a $30-\mu$-Dish (ibidi GmbH, Germany), and after $24 \mathrm{~h}$ the intact cells were visualized before and after treatment with anticancer drugs. Fluorescence lifetime images were analyzed by SymphoTime analysis software (PicoQuant $\mathrm{GmbH}$, Berlin, Germany). The amplitude weighted fluorescence lifetimes or mean fluorescence lifetimes $\left(\tau_{m}=\frac{a_{1} \tau_{1}+a_{2} \tau_{2}}{a_{1}+a_{2}}\right)$ were determined from the nonlinear least-square fitting of the decay curves. While single-exponential model was used to fit the decays of noninteracting donors, biexponential model was employed to fit the decay curves in case of interacting molecules as it is usually done in FLIMFRET experiments. ${ }^{63}$ The goodness of fit (chi-square) served as a guide for selection of the appropriate decay model.

\subsection{Animals}

Female 12-weeks-old BALBc/nude mice (Animal Breeding Center, Pushchino, Russia) of 22 to $25 \mathrm{~g}$ weight were used for tumor cell inoculation. All experimental procedures were approved by the local ethical committee for animal experiments. Animals were treated humanely. All of the subcutaneous inoculations and tumor injections were administered with regard for alleviation of suffering. Mice were checked weekly for the development of tumors and for fluorescence signal. For fluorescence measurements, animals were anesthetized intramuscularly with a 50- $\mu 1$ mixture of Zoletil 50 (Virbac, France) and Rometar $2 \%$ (Spofa Praha, Czech Republic) in equal proportion.

Cells for inoculation were harvested by trypsin treatment and washed in HEPES, $2 \times 10^{6}$ cells were suspended in $0.1 \mathrm{ml}$ of Dulbecco's phosphate buffer saline, inoculated dorsally in nude mice, and analyzed twice a week for fluorescence growing [Fig. 2(a)]. At indicated time points, the length, height, and width of tumors were measured by caliper. Tumor volumes $(V)$ were calculated according to

$V=\pi / 6 *(D 1 \times D 2 \times D 3)$,

where $D 1, D 2$, and $D 3$ are length, height, and width of the tumor in millimeters, respectively.

On the seventh day of growth, anticancer therapy was applied. For fluorescence measurements, animals were anesthetized intramuscularly with a 50- $\mu$ l mixture of Zoletil 50 (Virbac, France) and Rometar 2\% (Spofa Praha, Czech Republic) in equal proportion.

All fluorescence measurements were performed using transdermal configuration for excitation and emission on a whole intact animal. Skin flap, glass window, or any clearance procedure was not used.

Taxol (paclitaxel Lance, Russia) was introduced i.v. at a dose of $15 \mu \mathrm{g} / \mathrm{kg}$ for 5 days. Fluorescence was measured twice a week. Etoposide-Teva (Teva, The Netherlands) and 


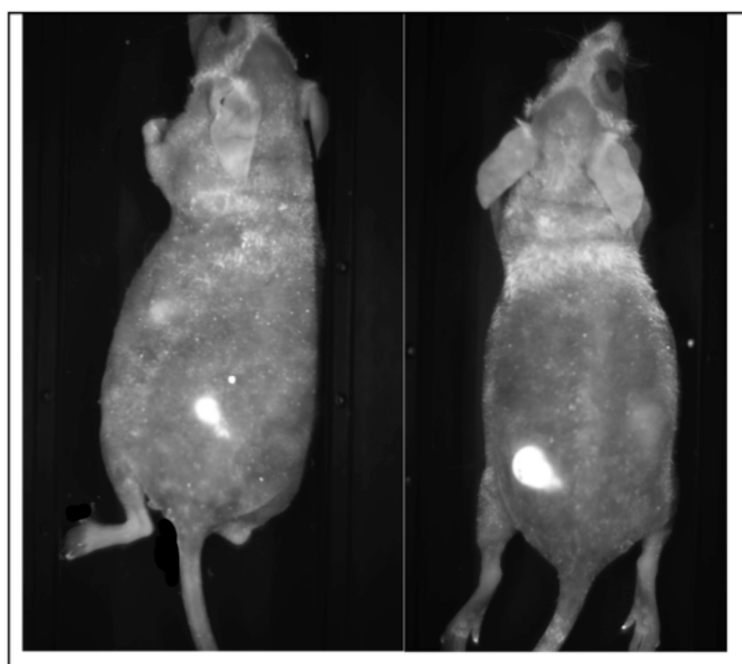

(a)

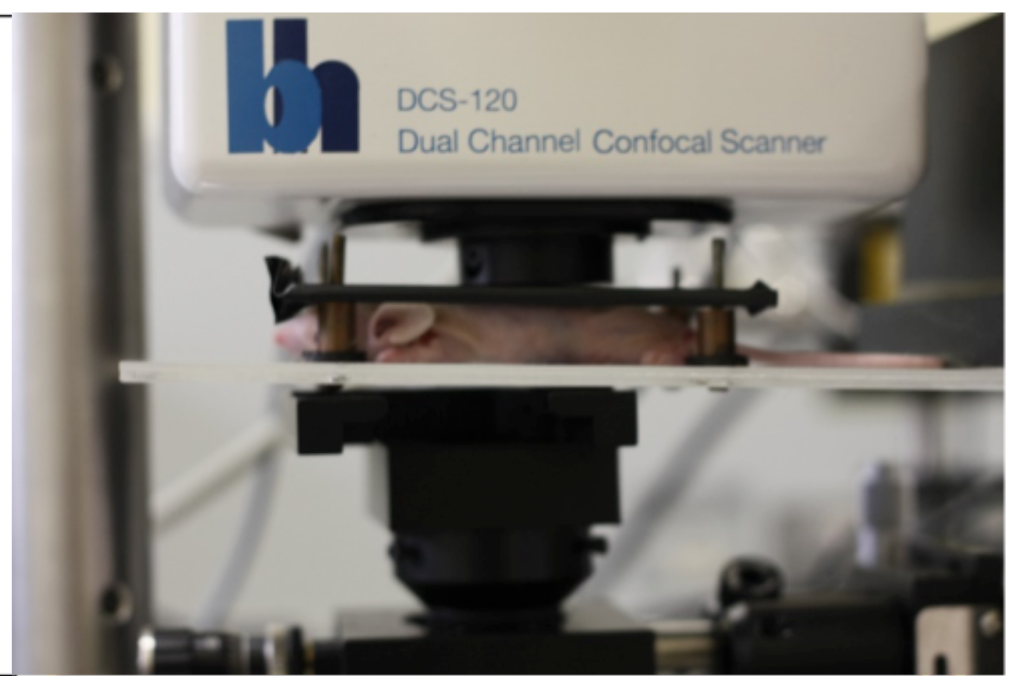

(b)

Fig. 2 (a) 2-D planar fluorescence image of mice bearing tumors A549-TR23K before (left image) and after 7 days of Taxol treatment (right image) (iBox, ex. 502 to $547 \mathrm{~nm}$, em. 580 to $640 \mathrm{~nm}$ ); (b) FLIM measurements of intact anesthetized mouse using DCS-120 confocal scanner.

cisplatin-Teva (Teva, Netherlands) were injected i.p. at a dose of 5 and $5 \mu \mathrm{g} / \mathrm{kg}$ for 5 days $i . v$, respectively.

\subsection{Local Fluorescence Spectroscopy}

The fluorescence spectra of the tumor xenografts were measured using a fiber optical spectrometer FS-003V spectrometer (CLUSTER Ltd., Russia). Fluorescence was excited by 532-nm diode-pumped solid-state laser in CW mode. The typical exposure times was $50 \mathrm{~ms}$. A Y-shaped ring fiber bundle (seven fibers with a diameter of $100 \mu \mathrm{m}$ and a numerical aperture of 0.22 ) was used to deliver the excitation laser radiation to the tissue (a central fiber) and to transmit the fluorescence radiation to the photodetector (six peripheral fibers). The laser power transmitted by the fiber was $\sim 5 \mathrm{~mW}$. The spatial resolution was 0.3 to $1 \mathrm{~mm}$ in the surface measurements. From three to five spectra were measured for each sample and processed using specialized software (LightView, CLUSTER Ltd., Russia).

\subsection{2-D Planar Imaging}

2-D planar fluorescent images of anesthetized mouse were acquired in epifluorescence mode using the iBox imaging system (UVP) equipped with a 16-bit monochrome-cooled CCD camera (BioChemi HR Camera, UVP) and a 150-W halogen quartz lamp (BioLite, UVP). Animal plate was equipped by motorized platform for magnification and focusing. Measurements were performed using bandpass excitation (502 to $547 \mathrm{~nm}$ ) and emission filters (570 to $640 \mathrm{~nm}$ ). Images were obtained at various time points of tumor growth using vision works image acquisition, acquisition time was $60 \mathrm{~s}$. Images were analyzed using vision works analysis software [Fig. 2(a)].

\subsection{FLIM-FRET of Tumor Xenografts}

The fluorescence lifetime images of tumor xenografts were acquired on a macroscopic DCS-120 confocal scanning system (Becker \& Hickl GmbH, Berlin, Germany) equipped with 532-nm picosecond diode laser PicoTA (PicoQuant GmbH, Berlin, Germany) (repetition rate and pulse-width are $80 \mathrm{MHz}$ and $100 \mathrm{ps}$, respectively) and HPM-100-40 hybrid detector (Becker \& Hickl GmbH, Berlin, Germany). Animals were put in a cassette on a mobile stage [Fig. 2(b)]. Fluorescence emission from a tumor was collected through the skin in the epi-illumination configuration.

The maximum diameter of the image area in the primary image plane of the scanner is about $15 \mathrm{~mm}$. The size of the laser spot in the image plane is estimated to be $15 \mu \mathrm{m}$. The average laser power incident on the sample is about $50 \mu \mathrm{W}$. This resulted in energy density of $0.7 \mu \mathrm{J} / \mathrm{cm}^{2}$, which is well below the damage threshold for biological samples. The numerical aperture of the detection beam is given by the beam diameter (about $3 \mathrm{~mm}$ ) and the focal length of the scan lens $(40 \mathrm{~mm})$. The collection efficiency is thus considerably lower than in case of a microscope. However, macroscopic imaging can use much higher laser power, which compensates for low collection efficiency. To separate excitation and fluorescence signals long- and bandpass filters were used (HQ550LP and 580BP, Chroma). Image collection time for anesthetized mouse varied from 2 to 5 min depending on the level of fluorophores expression. Lifetime images have been analyzed with SPCImage 3.2 data analysis software (Becker \& Hickl GmbH, Berlin, Germany). Standard deviations were calculated using SPCImage 3.2 data analysis software.

\section{Results}

\subsection{FLIM of Transduced Tumor Live Cells In Vitro}

Lentiviral transduction of A549 and HEp-2 cells with the TR23K caspase-3 sensor plasmid was accomplished with $70 \%$ to $90 \%$ efficiency. ${ }^{53}$ This high efficiency allowed us to bypass the stages of selection and cloning while maintaining the initial heterogeneity of cell lines. ${ }^{64}$ The cell lines obtained demonstrated a high level of expression of the sensor during the whole time of maintenance. 


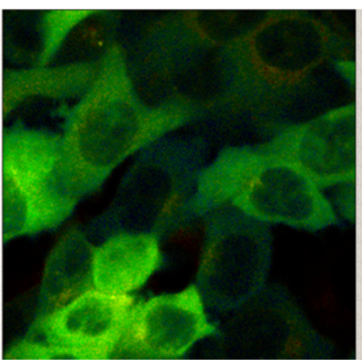

Control

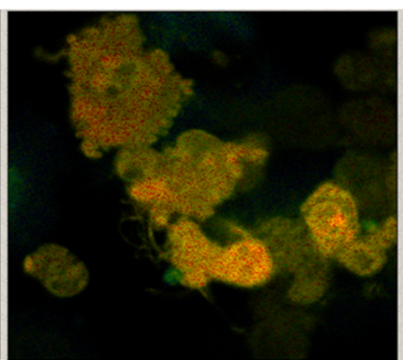

cisplatin

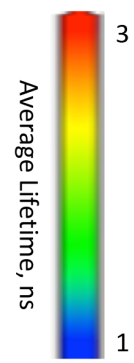

1

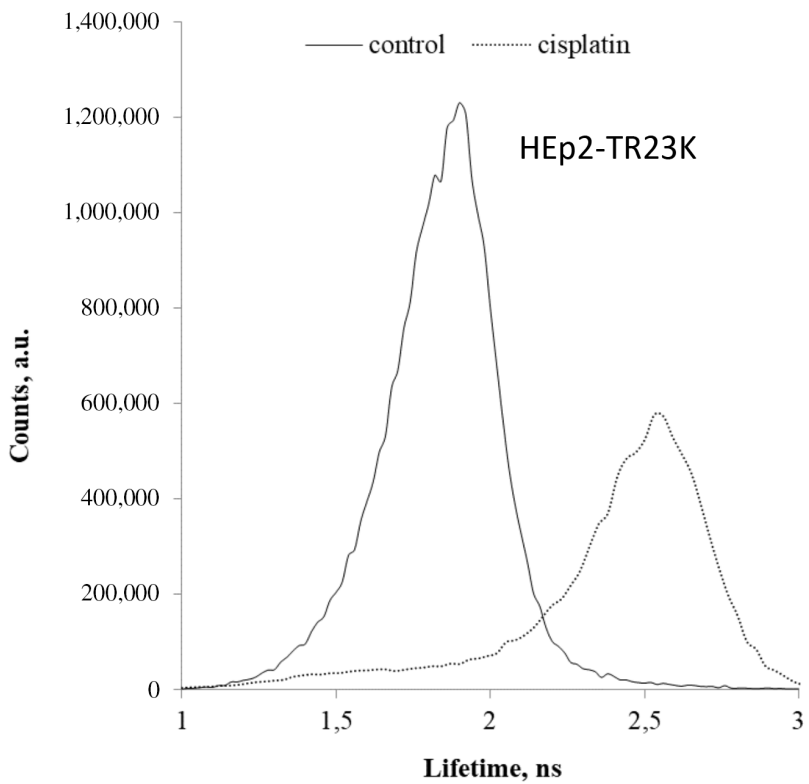

(a)

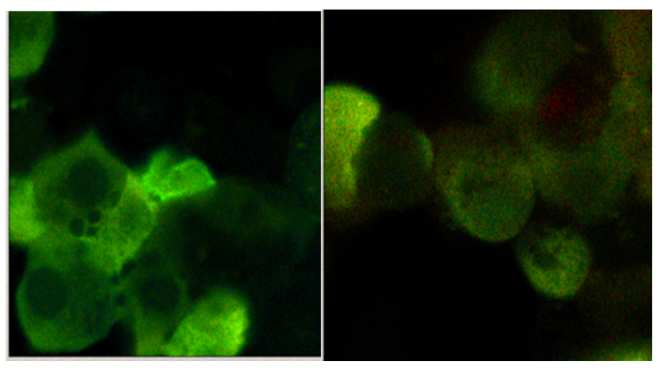

paclitaxel

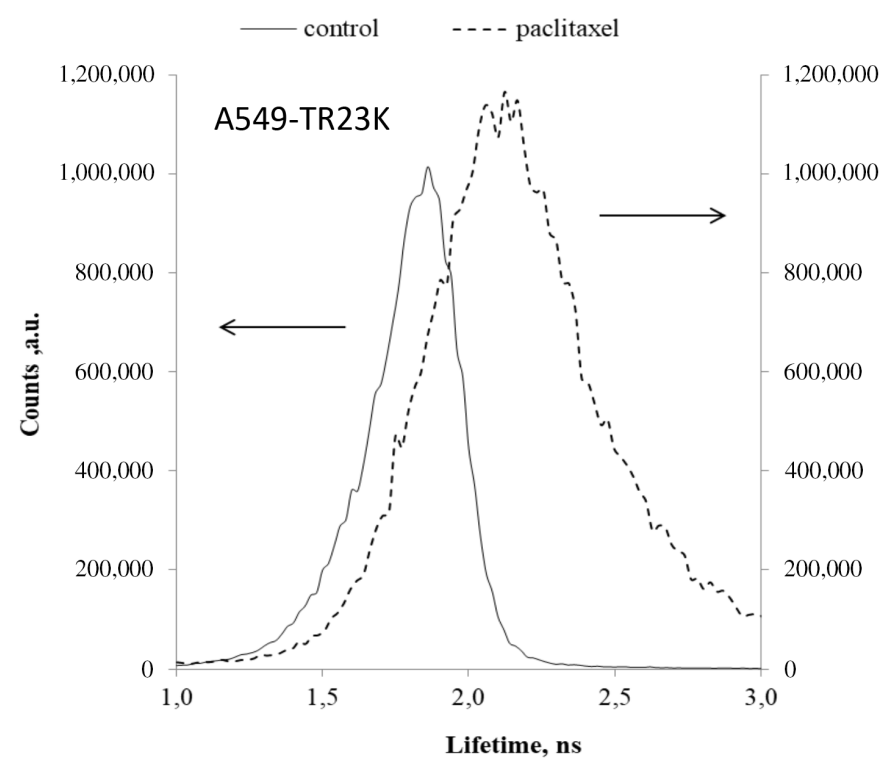

(b)

Fig. 3 (a) FLIM of HEp-2-TR23K and corresponding lifetime distribution before (left image, solid line on the histogram) and after $24 \mathrm{~h}$ of treatment with $200 \mu \mathrm{g} / \mathrm{ml}$ of etoposide and $10 \mu \mathrm{g} / \mathrm{ml}$ of cisplatin (right image, dot line on the histogram); (b) FLIM of A549-TR23K and corresponding lifetime distribution before (left image, solid line on the histogram) and after $24 \mathrm{~h}$ of treatment with $30 \mu \mathrm{g} / \mathrm{ml}$ of paclitaxel (right image, dash line on the histogram). Image size $80 \times 80 \mu \mathrm{m}$. (MicroTime 200, objective UPlanSApo $60 \times / 1.35)$

FLIM-FRET of transduced A549-TR23K and HEp-2TR23K cells was performed using confocal time-resolved microscopy. Etoposide, cisplatin, and paclitaxel were used in the range of doses causing cytotoxicity in $30 \%$ to $90 \%$ of cells after $24 \mathrm{~h}$ of treatment (data not shown). Caspase-3 activity in cells was estimated by the shift in a lifetime distribution histogram from 1.8 to $1.9 \mathrm{~ns}$ (lifetime of intact sensor) up to the $2.5 \mathrm{~ns}$ (lifetime of cleaved sensor) as was demonstrated earlier. ${ }^{53}$

Results on lifetime distribution analysis in HEp-2-TR23K differed from those in A549-TR23K. While HEp-2-TR23K have exhibited the sensitivity to cisplatin and etoposide, A549-TR23K proved to be sensitive only to paclitaxel. In this way, the cleavage of the caspase sensor caused by cisplatin and etoposide in the HEp-2-TR23K cells, and by paclitaxel in the A549-TR23K cells has been demonstrated in vitro [Figs. 3(a) and 3(b)], respectively. I was shown that these anticancer drugs could promote an activation of caspase-3 in doses and regimes suitable for in vivo study as it was shown earlier. ${ }^{65-67}$ So it was expected that tumor xenografts based on TR23K-transduced cells would demonstrate the same specific responses to treatment with anticancer drugs described already.

\subsection{FLIM and Steady-State Measurements of Tumor Xenografts In Vivo}

Tumor xenografts of A549-TR23K and HEp-2-TR23K were based on the same cells expressing the caspase-3 sensor. Control tumor xenografts A549-TagRFP and HEp-2-TagRFP were based on cells expressing TagRFP only, thus imitating the ideal situation of total cleavage of the sensor under similar physiological conditions. Fluorescence measurements were performed after establishing of xenografts, which took about 2 weeks. It should be noted that xenografts based on A549 cells were of highly fluorescence and characterized by nodular and expansive tumor growth. The other xenograft model using HEp- 2 cells exhibited weak fluorescence and characterized by both surface growth and tissue penetration.

The monitoring of fluorescence of tumor xenografts has been done by local fluorescence spectroscopy and 2-D planar imaging by $\mathrm{iBOX}$ as well as lifetime imaging by confocal laser scanner DCS-120. In control experiments, it has been shown that the change in fluorescence intensity was proportional to increasing tumor volume of A549-TagRFP and HEp-2-TagRFP xenografts during the initial stage of growth (data not shown). 


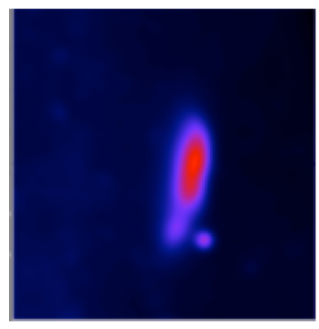

8th day

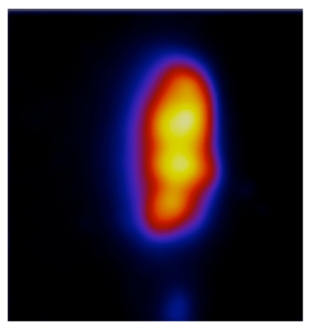

16 th day

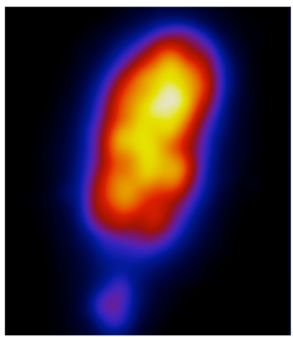

25th day

(a)

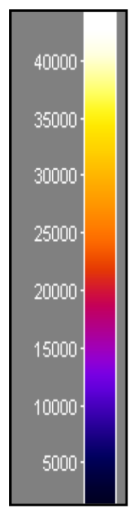

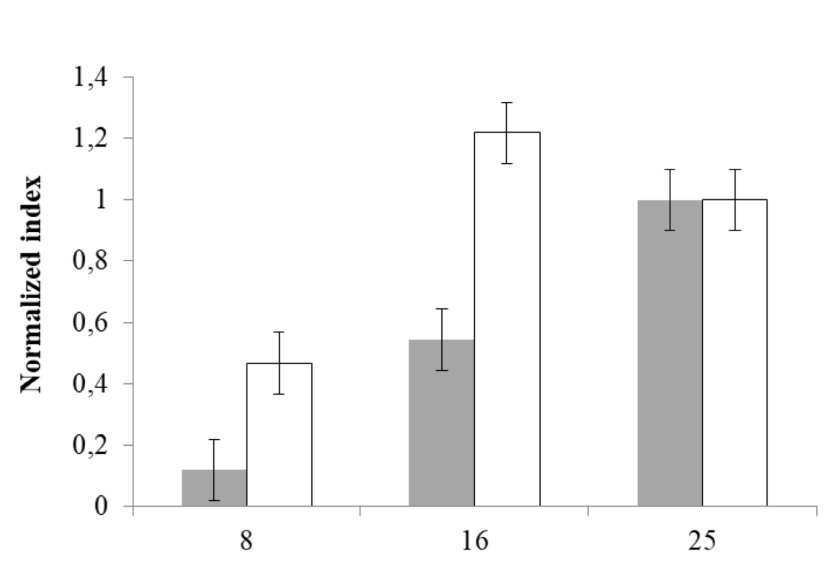

Day of tumor growth

(b)

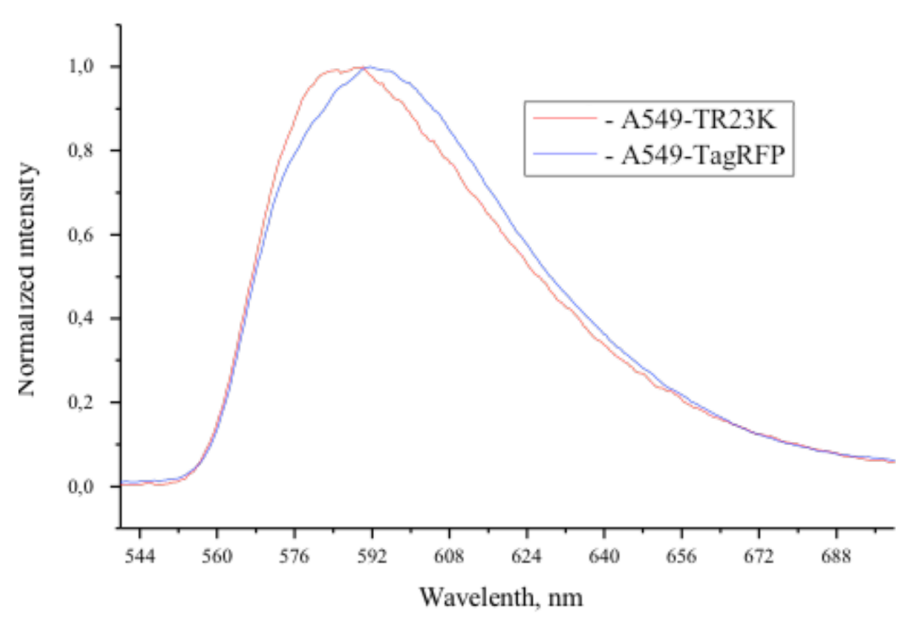

(c)

Fig. 4. Fluorescence of tumor xenografts A549-TR23K on 8th, 16th, 25th day of tumor growth: (a) pseudocolor mapping of the intensity of tumor fluorescence (iBox, ex 502 to $547 \mathrm{~nm}$, em 580 to $640 \mathrm{~nm}$ ). (b) Fluorescence of the tumor xenograft A549-TR23K (white) and the tumor size (gray) normalized to the 25th day of growth on 8th, 16th, 25th day of tumor growth. ROI of tumor fluorescence were depicted manually using Image $\mathrm{J}(\mathrm{NIH})$. The same ROI were moved to the nonfluorescent skin region. Fluorescence contrast was calculated as a relationship of integral fluorescence of tumor to integral fluorescence of skin. Normalized index was calculated as a ratio of fluorescence contrast on indicated dates to the fluorescence contrast at the last date of measurements (25th day). (c) Spectra of tumor xenografts A549-TR23K and A549-TagRFP (FS-003V spectrometer, ex. $532 \mathrm{~nm}$ ).

For xenografts expressing the TR23K biosensor, the relation to the increase of tumor volume was not reproduced in a consistent manner [Fig. 4(b)]. Spectra of xenografts expressing the TR23K sensor with the maximum of fluorescence at $585 \mathrm{~nm}$ did not show a significant shift compared to control xenografts expressing only TagRFP with the maximum of fluorescence at $595 \mathrm{~nm}$ [Fig. 4(c)].

Figure 5 demonstrates the diversity of tumor-to-skin fluorescence intensity ratio obtained by planar imaging and lifetimes of xenografts expressing the TR23K sensor, only TagRFP, and without any FPs. The fluorescence intensity distribution was more diverse compared to lifetime readouts.

It should be noted that xenografts based on A549 cells were of highly fluorescence and characterized by nodular and expansive tumor growth. The other xenograft model using HEp-2 cells exhibited weak fluorescence and characterized by both surface growth and tissue penetration. Tumor to skin ration of fluorescence in A549-based tumors and HEp-2-based tumors differed three to five times according to Fig. 5.

The relationship among fluorescence intensity, lifetime, and tumor volume of xenografts expressing control TagRFP is presented in Fig. 6. The most significant feature is that the lifetime of tumor increases on initial stage of growing and approaching the value of 2400 to 2500 ps which corresponds to the lifetime of TagRFP in vitro.

\subsection{Lifetime Imaging in Xenografts Based on the HEp-2 Tumor Cells}

Here, we presented the analysis of FLIM and lifetime distribution in one set of experiments. Four animals were monitored and treated simultaneously from the 0 to 32 nd days of xenografts stabilization and growing. The mean fluorescence lifetime was determined from one/two exponential fit of the decay curve.

Mean lifetime of nontreated xenograft HEp-2-TR23K on 17 th day of growth was of $1679 \pm 99$ ps [Fig. 7, images (a) with corresponding histograms $\left(\mathrm{a}^{\prime}\right)$ ]. Lifetime distribution in treated HEp-2-TR23K xenograft changed to $1957 \pm 110 \mathrm{ps}$ on the 7th day after chemotherapy's start (23rd day of tumor growth) [Fig. 7, image (b) with corresponding histogram $\left(b^{\prime}\right)$ ]. Lifetime of another nontreated tumor xenograft within the same terms of growth (23rd day of tumor growth) was estimated as 


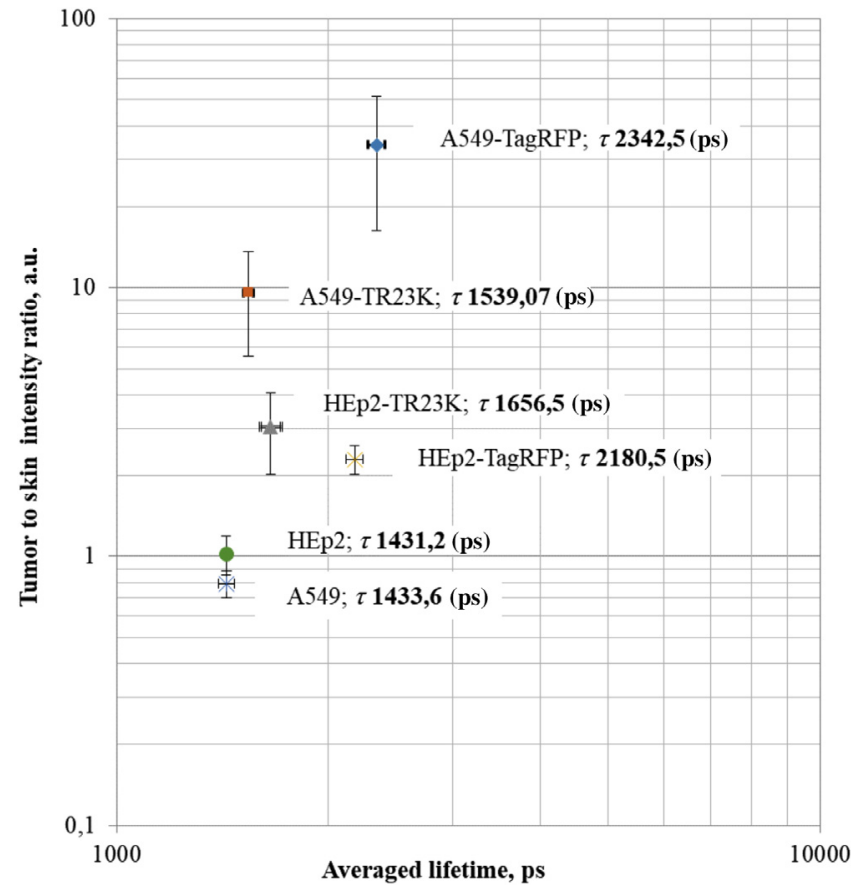

Fig. 5 Distribution of mean fluorescence intensity versus lifetimes for tumor xenografts at different stages of growth (from 8 to 32 days), $\tau$, mean lifetime ( $n=6$ to 10 ) with calculated custom standard deviation.

$1830 \pm 114$ ps [Fig. 7, images (c) with corresponding histograms $\left.\left(c^{\prime}\right)\right]$. We observed that the stage of tumor growth correlated with an increase in lifetime. In control experiment with HEp-2-TagRFP xenograft that simulates totally split sensor, the mean lifetime was $2212 \pm 59 \rho$ s within the same terms. Remarkable heterogeneity of TagRFP lifetime distribution in this control xenograft was noticed, most probably due to the different depth and specificity of tumor invasion as presented in

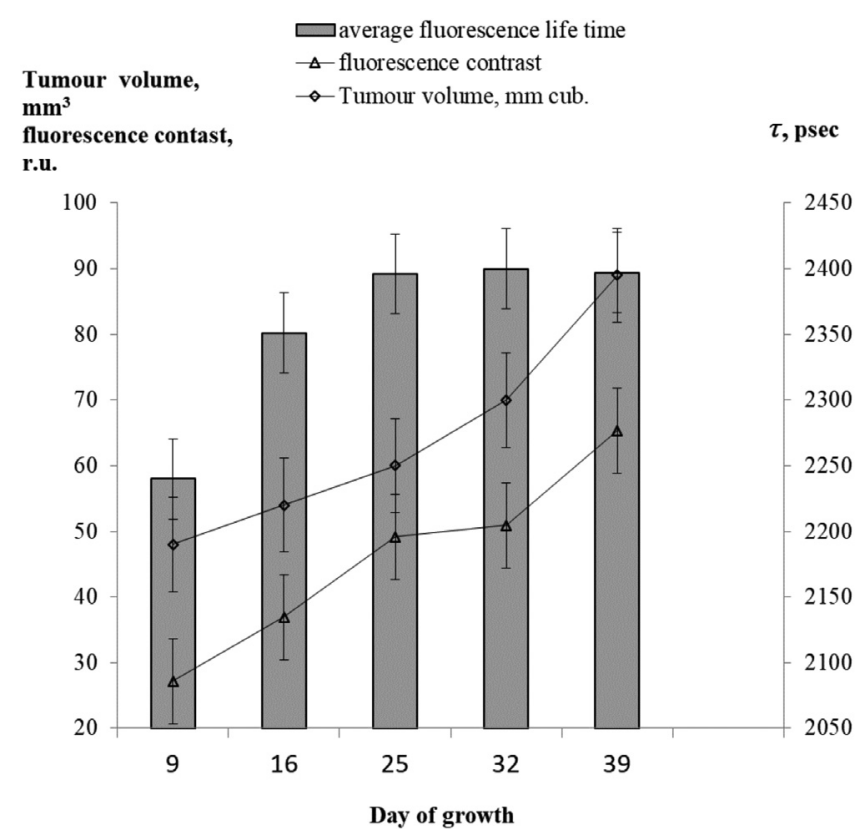

Fig. 6 Relationship between tumor volume and average fluorescence intensity and lifetime for A549-TagRFP. the corresponding image [Fig. 7, image (d) with corresponding histogram $\left.\left(d^{\prime}\right)\right]$.

\subsection{Lifetime Imaging in Xenografts Based on the A549 Tumor Cells}

The mean lifetime in highly fluorescent A549-TR23K sensorexpressing xenografts starts from $1672 \pm 174$ ps on the 16th day [Fig. 8, images (a) with correspondent histogram $\left(a^{\prime}\right)$ ]. On the 7th day after chemotherapy the distribution became bimodal, the component of $1700 \pm 106 \rho$ s retained [Fig. 8, image (b) with corresponding peak $\left.\left(b^{\prime}\right)\right]$ and a peak of $2100 \pm$ 216 ps appeared [Fig. 8, image (b), area $\left(b^{\prime}\right)$, and histogram $\left.\left(b^{\prime \prime}\right)\right]$. The decay curve of A549-TR23K xenografts after treatment from the region of interest (ROI) was fitted with two exponential model $\left(\chi^{2}=1.1\right)$ (Fig. 9).

The fluorescence of nontreated A549-TR23K xenograft did not change significantly within a week, it was measured as $1604 \pm 213$ ps on 23rd day [Fig. 8 image (c) with corresponding histogram $\left(\mathrm{c}^{\prime}\right]$ under the same conditions of animal maintenance. The mean lifetime of control xenograft A549-TagRFP, imitating total cleavage of the sensor, was $2400 \pm 49$ ps [Fig. 8, image (d) with corresponding histogram $\left(\mathrm{d}^{\prime}\right]$ and is very narrow. The difference of lifetime distribution of the same FP TagRFP and sensor TR23K in xenografts bearing A549 and xenografts bearing HEp-2 could be connected with individual characteristics of cell lines and features of growth of xenografts. We presumed that every animal bore only one tumor.

The study of the tumor models allowed us to determine that the anticancer treatment led to the shifts of mean lifetime distribution in both types of xenografts. In case of A549-TR23K, the bimodal distribution lifetime signal [Fig. 8, image (b) with corresponding histogram] supported the idea of heterogeneous responses of a tumor to chemotherapy. ${ }^{68,69}$ In the case of HEp-2TR23K, the shift was not so pronounced and with a single mode which coincided with a more homogeneous response of the tumor to chemotherapy.

\section{Discussion}

Here, we compared two approaches of evaluating caspase-3 activity in vivo (in live animals) using the genetically encoded FRET-sensor TR23K proposed earlier. ${ }^{51,52}$ Planar imaging of xenografts without drug treatment demonstrated the high level of intensity dispersion independent of cell type (Fig. 5). This dispersion could possibly be due to the different expression level of genetically encoded FRET-sensors and microheterogeneity of tumor. Moreover, as shown in Fig. 4(b), the spectral shift between the intact and cleaved form of the sensor, as judged by the TagRFP emission spectrum is too small for spectral resolution.

Only the FLIM-FRET method provided the possibility to image caspase- 3 activation and estimate the heterogeneity of the response to chemotherapy in different regions of tumors. Earlier the FLIM-FRET analysis of caspase-3 activity using TR23K sensor was performed in tumor cells and caspase-3-dependent apoptosis was demonstrated. ${ }^{53}$ In this work, visualization of caspase- 3 activation in subcutaneous tumor xenografts was accomplished using a confocal time-resolved macroimaging scanning system. Tumor xenografts expressing only TagRFP were used as a control and provided the lifetimes of the donor fluorophore in the FRET-pair under the same conditions in vivo in the case of total cleavage of the sensor. 


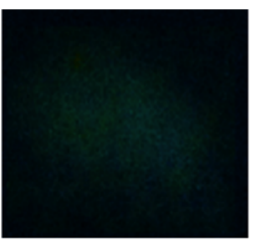

(a)

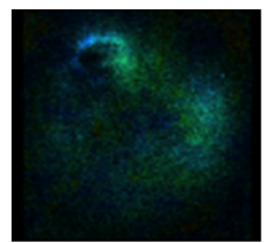

(b)

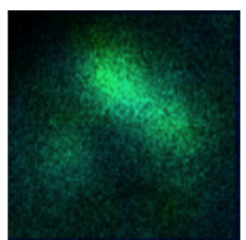

(c)

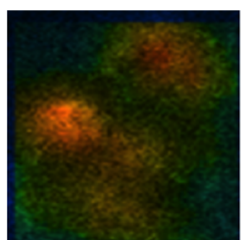

(d)

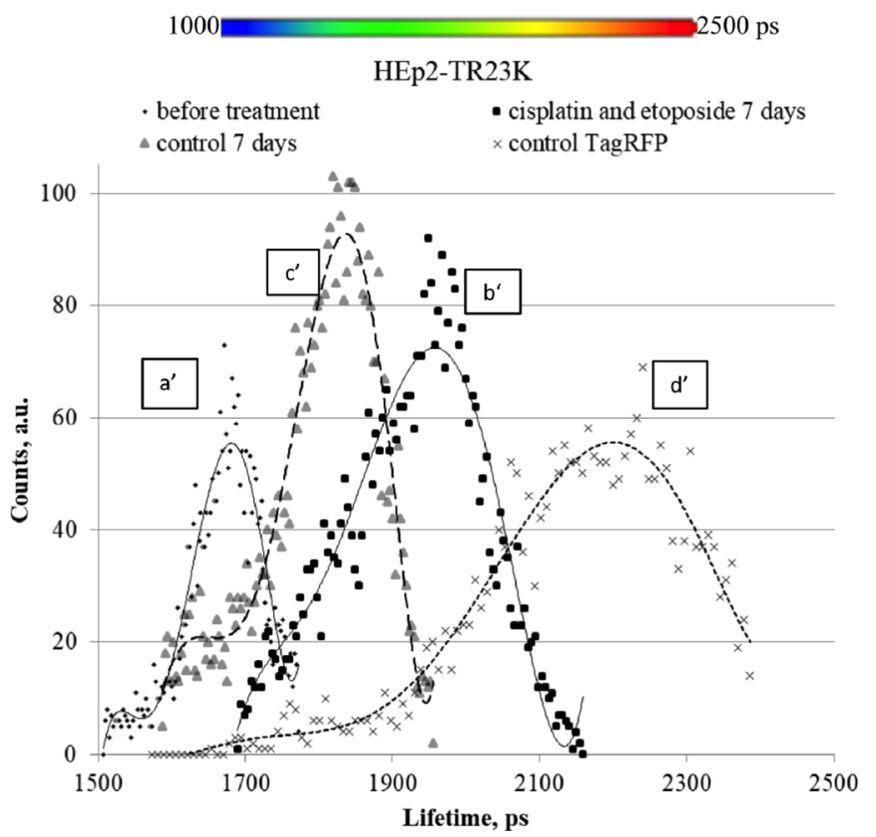

(e)

Fig. 7 FLIM-FRET of tumor xenografts based on the larynx carcinoma HEp2 (a-d, upper images), pseudocolor scale bar (e) and corresponding lifetime distribution histograms: (a) HEp2-TR23K before treatment with cisplatin and etoposide with corresponding histogram (a') (before treatment); (b) HEp2-TR23K on the 7th day of treatment with cisplatin and etoposide with corresponding histogram ( $\left.b^{\prime}\right)$ (cisplatin and etoposide 7 days); (c) HEp2-TR23K control with correspondence histogram (c') (control 7 days); (d) HEp2-TagRFP with corresponding histogram ( $d^{\prime}$ ) (control TagRFP); (e) pseudocolor scale bar from 1000 to 2500 ps.

Administration of anticancer agents led to cleavage of the sensor that was confirmed by lifetime distribution shifts to longer lifetimes in vivo for both tumor xenograft models. The A549-TR23K xenografts demonstrated a bimodal distribution of lifetimes. One of the components corresponds to the lifetime of the intact form of the sensor in vivo, while the long-live shoulder was close to the distribution of free TagRFP which imitated total cleavage of the sensor. The large intact pool of the sensor in case of treated A549-TR23K xenograft [Fig. 8, image (b) with corresponding histogram] also supports the observation that this tumor exhibits abnormal behavior after treatment with Taxol. ${ }^{70}$

Heterogeneity of anticancer treatment response in tumor models may be associated with features of the pharmacokinetics of anticancer agents in tumor xenografts. This may depend on the growth stage of the tumor and on the characteristics of tumor blood supply. ${ }^{71}$ The role of these parameters requires further investigation. Other possible factors whose roles need to be determined are spontaneous apoptosis in growing tumors ${ }^{72}$ or a form of reversible transient caspase- 3 activation. ${ }^{29}$

The average lifetime values of biosensor and free-TagRFP fluorescence in different types of xenografts in vivo did not correspond to the in vitro (cuvette) measurements, 1600 to $1800 \mathrm{ps}$ and 2200 to 2400 ps (this paper) against the 1800 and 2500 ps for the TR23K and TagRFP in cuvette, respectively. ${ }^{52} \mathrm{We}$ also observed that the fluorescence lifetime of TagRFP in tumor xenografts increased with tumor growth [days 7 to 23, Figs. 7(a) and 7(c)]. We did not observe any significant autofluorescence signals that could impair fluorescence lifetime measurements at the excitation wavelength. The autofluorescence signal (data not shown) of nonfluorescent tumor (without any sensors or FPs) was $\sim 50$ to 100 times lower than the signal from a fluorescent tumor in the same detection window $(580 \pm 20 \mathrm{~nm})$.

Differences between in vivo and in vitro measurements in live cells can be explained by two groups of factors. First of all, it may be different optical surrounding of sensor. The primary lifetime data measured on live animal/cells should be classified as "a probabilistic data" (or appeared data) that dependent on multiple photon scattering and refractive index and, consequently, it should depend on different stages of cell cycle or apoptosis, on tumor grows/size and localization in animal body. The role of refractive index in microscopic lifetime measurements is discussed in Ref. 50. The problem of the multiple photon scattering can be solved by the fluorescence lifetime tomography. ${ }^{33}$

In addition to aforementioned pharmacokinetics factors, another group of factors of pure biological origin are typical for live animals or cell populations. In our research, genetically 


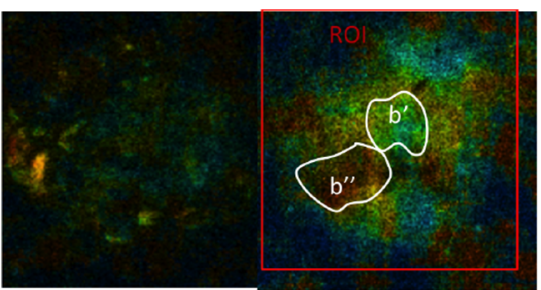

(a) (b)

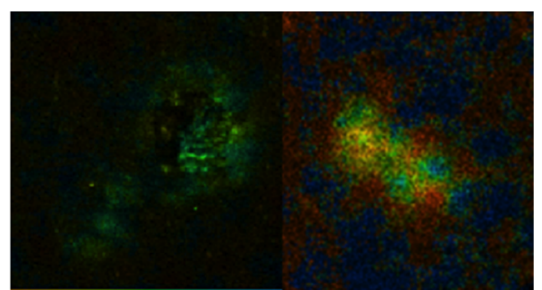

(c) (d) 1000 $2500 \mathrm{ps}$

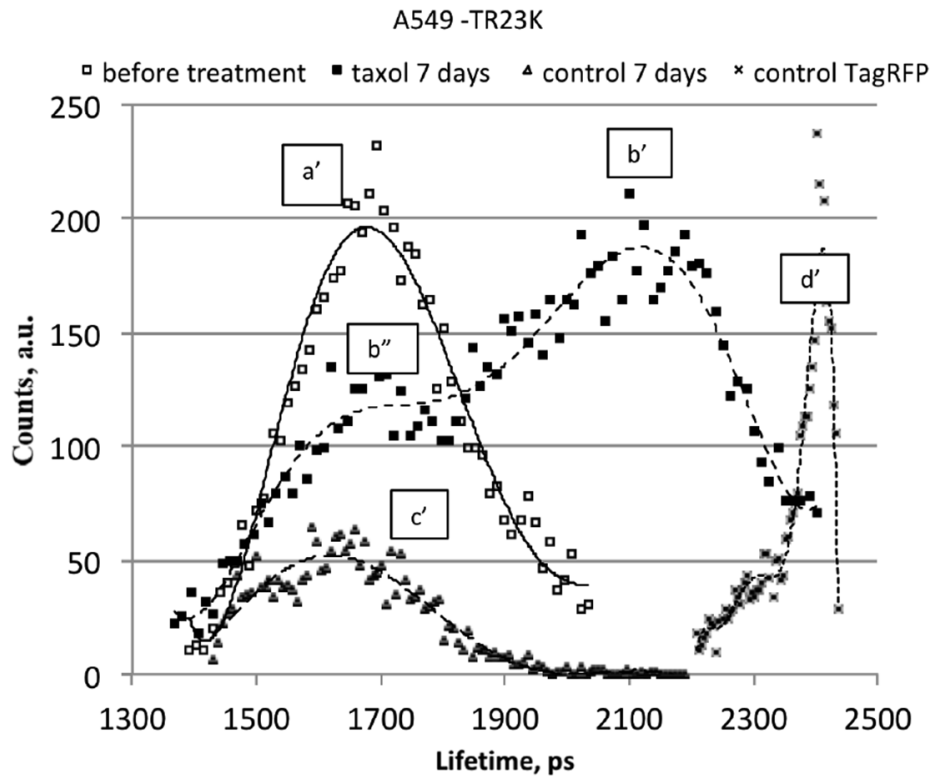

(e)

Fig. 8 FLIM-FRET of tumor xenografts based on the lung adenocarcinoma A549 (a-c, upper images) and corresponding lifetime distribution (bottom images): (a) A549-TR23K before treatment with Taxol (16th day of growth) with the corresponding histogram (a' (before treatment); (b) A549-TR23K on the 7th day of treatment with Taxol (23rd day of growth) with the corresponding two peak histogram, short-lived ( $b^{\prime}$ and long-lived $\left(b^{\prime \prime}\right)$ (Taxol 7 days). The areas of short-lived $\left(b^{\prime}\right)$ and long-lived $\left(b^{\prime \prime}\right)$ components of cleaved sensor are bounded by white line at the image (b); (c) A549-TR23K control, (23rd day of growth) with the corresponding histogram ( $c^{\prime}$ (control 7 days); (d) A549-TagRFP, (23rd day of growth) with corresponding histogram ( $\mathrm{d}^{\prime}$ (control TagRFP); (e) pseudocolor scale bar from 1000 to $2500 \mathrm{ps}$.

encoded FRET-sensors are under constitutive promoter ${ }^{51,52}$ and are permanently synthesized de novo on ribosomes according to the cell cycle program, because this promoter control expression in the cell and are independent of environmental and developmental factors. After induction of caspase- 3 activity by certain drugs during the course of measurements, the resulting lifetime distribution histogram should contain overlap of cleaved and de novo synthesized sensors and peak position of the histogram or bimodal distribution should depend on the efficiency of these two processes as well as a general speed of cell grows in solid tumor. Moreover, cells are nonsynchronized in cell cycle in solid tumor models that can lead to the manifestation of different stages of apoptosis as well as different level of caspase-3 activity. All these parameters lead to uncertainty in precise peak position for lifetime distribution histogram, but continuous measurement of the same live animal during several weeks allow us to make correct conclusion concerning drug efficiency.

Thus, we show that the FLIM-FRET approach can be used as a tool for screening anticancer agents for their efficacy toward key molecular targets. Monitoring can be performed within a tumor during long period of observation, in this case during several weeks. The advantage of this assay is the ability to account for the individual characteristics of tumors. This approach can be used for the selection of anticancer agents and regimes as well as the study of the heterogeneity of cellular responses to therapy.

\section{Conclusions}

We demonstrated the possibility of visualizing caspase-3 activity using the FRET-sensor TR23K, which is based on a pair of proteins - a red FP as a donor and a chromoprotein as an acceptor. The in vivo assay is noninvasive and could be applied in strongly and weakly fluorescent subcutaneous xenografts in mice using the FLIM-FRET method.

Notably, our method provides the possibility to determine caspase-3 activation noninvasively through the whole period of tumor visualization for the same animal during several weeks without any surgical manipulations. This approach to individual monitoring is suitable for the estimation of antitumor responses individually on the same animal. This approach can 


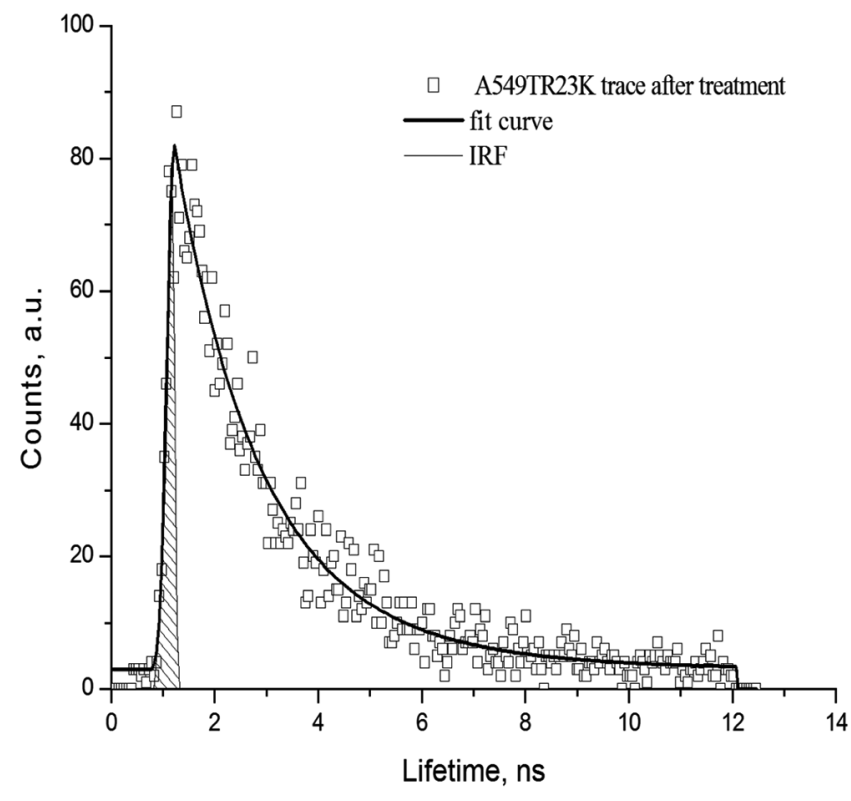

Fig. 9 Time trace in A549-TR23K xenograft after treatment with Taxol calculated from the ROI (red) of image from Fig. 8(b), IRF-instrument response function. Binning $\times 10, \chi^{2}=1.1$.

also be utilized in drug screening facilitating the development of new anticancer agents as well as improvement of chemotherapy and its adaptation for personal treatment.

\section{Disclosures}

The authors have declared that no competing interest exists.

\section{Acknowledgments}

The study on cell culture, confocal microscopy, FLIM-FRET on cells was supported by the Russian Scientific Foundation, grant 15-14-30019. The FLIM-FRET study on animals was partly supported by the Russian Foundation for Basic Research (1408-01017, 14-04-01266). The authors thank Dr. Alexander Goryashchenko for the critical reading of the section on FLIM-FRET microscopy of the manuscript and Dr. Maria Khrenova for her help in Fig. 1 preparation.

\section{References}

1. R. Weissleder et al., "In vivo imaging of tumors with protease activated near-infrared fluorescent probes," Nat. Biotechnol. 17(4), 375-378 (1999).

2. V. Ntziachristos et al., "In vivo tomographic imaging of near-infrared fluorescent probes," Mol. Imaging 1, 82-88 (2002).

3. X. He et al., "An overview of recent advances in quantum dots for biomedical applications," Colloids Surf. B 124, 118-131 (2014).

4. J. Zhou et al., "Upconversion nanophosphors for small-animal imaging," Chem. Soc. Rev. 41(3), 1323-1349 (2012).

5. J. Key et al., "Nanoparticles for multimodal in vivo imaging in nanomedicine," Int. J. Nanomed. 9, 711-726 (2014).

6. P. R. Contag et al., "Bioluminescent indicators in living mammals," Nat. Med. 4(2), 245-247 (1998).

7. R. A Sharrock et al., "The phytochrome red/far-red photoreceptor superfamily," Genome Biol. 9(8), 230 (2008).

8. G. S. Filonov et al., "Bright and stable near infra-red fluorescent protein for in vivo imaging," Nat. Biotechnol. 29(8), 757-761 (2011).

9. W. L. Rice et al., "In vivo tomographic imaging of deep-seated cancer using fluorescence lifetime contrast," Cancer Res. 75(7), 1236-1243 (2015).
10. J. Y. Hwang et al., "A multimode optical imaging system for preclinical applications in vivo: technology development, multiscale imaging, and chemotherapy assessment," Mol. Imaging Biol. 14(4), 431-442 (2012).

11. P. Timpson et al., "Imaging molecular dynamics in vivo-from cell biology to animal models," J. Cell Sci. 124(17), 2877-2890 (2011).

12. D. M. Chudakov et al., "Fluorescent proteins and their applications in imaging living cells and tissues," Physiol. Rev. 90(3), 1103-1163 (2010).

13. T. Abe et al., "Reporter mouse lines for fluorescence imaging," Dev. Growth Differ. 55, 390-405 (2013).

14. R. E. Campbell et al., "A monomeric red fluorescent protein," Proc. Natl. Acad. Sci. U. S. A. 99, 7877-7882 (2002).

15. D. Shcherbo et al., "Bright far-red fluorescent protein for whole-body imaging," Nat. Methods 4(9), 741-746 (2007).

16. M. Yang et al., "Whole-body optical imaging of green fluorescent protein-expressing tumors and metastases," Proc. Natl. Acad. Sci. U. S. A. 97(3), 1206-1211 (2000).

17. R. M. Hoffman et al., "The multiple uses of fluorescent proteins to visualize cancer in vivo," Nat. Rev. Cancer 5, 796-806 (2005).

18. A. L. Rusanov et al., "Fluorescence resonance energy transfer between fluorescent proteins as powerful toolkits for in vivo studies," Laser Phys. Lett. 8(2), 91-102 (2011).

19. Y. Chen et al., "Protein localization in living cells and tissues using FRET and FLIM," Differentiation 71(9-10), 528-541 (2003).

20. S. Rajoria et al., "FLIM-FRET for cancer applications," Curr. Mol. Imaging 3(2), 144-161 (2014).

21. D.W. Piston et al., "Fluorescent protein FRET: the good, the bad, and the ugly," Trends Biochem. Sci. 32(9), 407-414 (2007).

22. K. Truong et al., "The use of FRET imaging microscopy to detect protein-protein interactions and protein conformational changes in vivo," Curr. Opin. Struct. Biol. 11, 573-578 (2001).

23. T. Nagai et al., "A variant of yellow fluorescent protein with fast and efficient maturation for cell-biological applications," Nat. Biotechnol. 20, 87-90 (2002).

24. H. Ai et al., "Fluorescent protein FRET pairs for ratiometric imaging of dual biosensors," Nat. Methods 5, 401-403 (2008).

25. N. C. Shaner et al., "A guide to choose fluorescence proteins," Nat. Methods 2(12), 905-909 (2005).

26. M. Z. Lin et al., "Autofluorescent proteins with excitation in the optical window for intravital imaging in mammals," Chem. Biol. 16(11), 11691179 (2009).

27. Y. Yang et al., "Molecular imaging of proteases in cancer," Cancer Growth Metastasis 2, 13-27 (2009).

28. B. Turk, "Targeting proteases: successes, failures and future prospects," Nat. Rev. Drug Discovery 5(9), 785-799 (2006).

29. H. L. Tang et al., "In vivo caspase tracker biosensor system for detecting anastasis and non-apoptotic caspase activity," Sci. Rep. 5, 9015 (2015).

30. S. Shalini et al., "Old, new and emerging functions of caspases," Cell Death Differ. 22(4), 526-539 (2015).

31. S. H. Bokman et al., "Renaturation of aequarea green-fluorescent protein," Biochem. Biophys. Res. Commun. 101, 1372-1380 (1981).

32. L. M. Felber et al., "Evaluation of the CFP-substrate-YFP system for protease studies: advantages and limitations," BioTechniques 36(5), 878-885 (2004).

33. J. McGinty et al., "In vivo fluorescence lifetime tomography of a FRET probe expressed in mouse," Biomed. Opt. Express 2(7), 1907-1911 (2011).

34. K. Takemoto et al., "Spatio-temporal activation of caspase revealed by indicator that is insensitive to environmental effects," J. Cell. Biol. 160, 235-243 (2003).

35. J. J.-H. Chiang et al., "Using co-cultures expressing fluorescence resonance energy transfer based protein biosensors to simultaneously image caspase-3 and $\mathrm{Ca}^{2+}$ signaling," Biotechnol. Lett. 27, 12191227 (2005).

36. M. Rehm et al., "Single-cell fluorescence resonance energy transfer analysis demonstrates that caspase activation during apoptosis is a rapid process. Role of caspase-3," J. Biol. Chem. 277, 24506-24514 (2002).

37. Y.-C. Hwang et al., "Use of fluorescence resonance energy transfer for rapid detection of enteroviral infection in vivo," Appl. Environ. Microbiol. 72(5), 3710-3715 (2006). 
38. J. Joseph et al., "High throughput ratio imaging to profile caspase activity: potential application in multiparameter high content apoptosis analysis and drug screening," PLoS One 6(5), e20114 (2011).

39. Y. Yamaguchi et al., "Live imaging of apoptosis in a novel transgenic mouse highlights its role in neural tube closure," J. Cell. Biol. 195(6), 1047-1060 (2011).

40. G. Zacharakis et al., "Fluorescent protein tomography scanner for small animal imaging," IEEE Trans. Med. Imaging 24(7), 878-885 (2005).

41. V. Ntziachristos et al., "Fluorescence molecular tomography resolves protease activity in vivo," Nat Med. 8, 757-761 (2002).

42. S. Bloch et al., "Whole-body fluorescence lifetime imaging of a tumor targeted near-infrared molecular probe in mice," J. Biomed. Opt. 10(5), 054003 (2005).

43. A. P. Savitsky et al., "Three-dimensional in vivo imaging of tumors expressing red fluorescent proteins," Methods Mol. Biol. 872, 97-114 (2012).

44. I. V. Turchin et al., "Fluorescence diffuse tomography for detection of red fluorescent protein expressed tumors in small animals," J. Biomed. Opt. 13(4), 041310 (2008).

45. A. Zelmer et al., "Noninvasive fluorescence imaging of small animals," J. Microsc. 252(1), 8-15 (2013).

46. V. Ntziachristos, "Going deeper than microscopy: the optical imaging frontier in biology," Nat. Methods 7(8), 603-614 (2010).

47. F. Leblond et al., "Preclinical whole-body fluorescence imaging: review of instruments. Methods and applications," J. Photochem. Photobiol B. 98(1), 77-94 (2010).

48. W. Becker, "Fluoresence life-time imaging-techniques and applications," J. Microsc. 247(2), 119-136 (2012).

49. Y. Sun et al., "Förster resonance energy transfer microscopy and spectroscopy for localizing protein-protein interactions in living cells," Cytometry, Part A. 83(9), 780-793 (2013).

50. K. Suhling et al., "Time-resolved fluorescence microscopy," Photochem. Photobiol. Sci. 4, 13-22 (2005).

51. J. R. Conway et al., "Developments in preclinical cancer imaging: innovating the discovery of therapeutics," Nat. Rev. Cancer 14(5), 314-328 (2014).

52. A. L. Rusanov et al., "Lifetime imaging of FRET between red fluorescent proteins," J. Biophotonics 3(12), 774-783 (2010).

53. A. P Savitsky et al., "FLIM-FRET imaging of caspase-3 activity in live cells using pair of red fluorescent proteins," Theranostics 2(2), 215-226 (2012).

54. H. Murakoshi et al., "A dark green fluorescent protein as an acceptor for measurement of Förster resonance energy transfer," Sci. Rep. 5, 15334 (2015).

55. A. Xiao et al., "Fluorescence lifetime imaging of apoptosis," Tomography 1(2), 115-124 (2015).

56. O. A. Zlobovskaya et al., "Genetically encoded far-red fluorescent sensors for caspase-3 activity," BioTechniques 60(2), 62-68 (2016).

57. A. Goryashchenko et al, "Detection of protease activity by fluorescent protein FRET sensors: from computer simulation to live cells," Methods Appl. Fluoresc. 6(2), 022001 (2017).

58. G. O. Fruhwirth et al., "Deep-tissue multiphoton fluorescence lifetime microscopy for intravital imaging of protein-protein interactions," Proc. SPIE 7183, 71830L (2009).

59. M. Keese et al., "Fluorescence lifetime imaging microscopy of chemotherapy-induced apoptosis resistance in a syngenic mouse tumor model," Int. J. Cancer 126(1), 104-113 (2010).

60. N. Takahashi et al., "Two-photon fluorescence lifetime imaging of primed SNARE complexes in presynaptic terminals and $\beta$ cells," Nat. Commun. 6, 8531 (2015).

61. N. Andrews et al., "Visualising apoptosis in live zebrafish using fluorescence lifetime imaging with optical projection tomography to map
FRET biosensor activity in space and time," J. Biophotonics 9(4), 414-424 (2016).

62. I. Shelaev et al., "The origin of radiationless conversion of the excited state in the kindling fluorescent protein (KFP): femtosecond studies and quantum modeling," Laser Phys. Lett. 8, 469 (2011).

63. H. Wallrabe and A. Periasami, "Imaging protein molecules using FRET and FLIM microscopy," Curr. Opin. Biotechnol. 16, 19-27 (2005).

64. S. J. Diaz-Cano, "Tumor heterogeneity: mechanisms and bases for a reliable application of molecular marker design," Int. J. Mol. Sci. 13, 1951-2011 (2012).

65. C. Dive et al., "Drug target interactions: only the first step in the commitment of a cell to a programmed cell death," $B r$. J. Cancer 64(1), 192-196 (1991).

66. L. Milas et al., "Combination of taxanes with radiation: preclinical studies," Semin. Radiat. Oncol. 9, 12-26 (1999).

67. A. R. Shoemaker et al., "A small-molecule inhibitor of Bcl-XL potentiates the activity of cytotoxic drugs in vitro and in vivo," Cancer Res. 66(17), 8731-8739 (2006).

68. G. H. Heppner et al., "Tumor heterogeneity: biological implications and therapeutic consequences," Cancer Metastasis Rev. 2(1), 5-23 (1983).

69. M. Jamal-Hanjani et al., "Translational implications of tumor heterogeneity," Clin. Cancer Res. 21(6), 1258-1266 (2015).

70. B. A. Weaver, "How Taxol/paclitaxel kills cancer cells," Mol. Biol. Cell 25(18), 2677-2681 (2014).

71. O. Tredan et al., "Drug resistance and the solid tumor microenvironment," J. Natl. Cancer Inst. 99, 1441-1454 (2007).

72. M.-B. Yin et al., "Involvement of cyclin D1-cdk5 overexpression and MCM3 cleavage in bax-associated spontaneous apoptosis and differentiation in an A253 human head and neck carcinoma xenograft model," Int. J. Cancer 83, 341-348 (1999).

Victoria Zherdeva is a senior research scientist of the Laboratory of Physical Biochemistry at the Federal Research Centre (fundamentals of biotechnology) of Russian Academy of Sciences. She graduated from Russian State Medical University. She received her PhD in biochemistry in 2007. Her research interests focus on experimental oncology and carcinogenesis, and investigation of metabolic feature of tumors using fluorescence imaging methods.

Natalia I. Kazachkina is a senior research scientist of the Laboratory of Physical Biochemistry at the Federal Research Centre (fundamentals of biotechnology) of Russian Academy of Sciences. She received her $\mathrm{PhD}$ in biology in 2003. Her research interests are the experimental oncology in particular anticancer therapy, photodynamic therapy, and fluorescence diagnostics.

Vladislav Shcheslavskiy, graduated with diploma from Physics Department, M.V. Lomonosov Moscow State University in 1993 and competed his PhD in physics in 2003 at the University of WisconsinMilwaukee. Currently, he is a senior research scientist at Becker \& Hickl GmbH. His research interests include fluorescence lifetime imaging, single molecule spectroscopy, and microscopy.

Alexander P. Savitsky, professor of biochemistry, graduated from the Chemistry Department of M.V. Lomonosov Moscow State University in 1974, received his PhD in 1979 and Doctor of Science in 1990 both in biochemistry from the same University. In 1991, he became the head of the Laboratory of Physical Biochemistry at the Federal Research Centre (fundamentals of biotechnology) of Russian Academy of Sciences. His research interests include fluorescence spectroscopy, microscopy, fluorescent proteins, in vivo imaging, molecular nanobiosensors, lanthanide, and phosphorescent labeling technology. 